\title{
Determination of Kerr and two-photon absorption coefficients of indandione derivatives
}

\author{
Arturs Bundulis, Igors Mihailovs, Edgars Nitiss, Janis Busenbergs, Martins Rutkis \\ Institute of Solid State Physics, University of Latvia, Kengaraga 8, 1068 Riga, Latvia
}

\begin{abstract}
We studied nonlinear optical properties of two different aminobenziliden-1,3-indandione derivatives - DDMABI and DMABI-OH by employing the Z-scan method. Through this we described how different donor and acceptor groups influence third-order nonlinear optical properties such as Kerr effect and two-photon absorption. During experimental measurements we used $1064 \mathrm{~nm} \mathrm{Nd}$ :YAG laser with $30 \mathrm{ps}$ pulse duration and $10 \mathrm{~Hz}$ repetition rate. From acquired values of Kerr and two-photon absorption coefficients we calculated values for real and imaginary parts of third-order susceptibility, as well as second-order hyperpolarizability. Quantum chemical calculations were carried out for secondorder hyperpolarizability to study how well calculations correlate with experimental values. Acquired data for DDMABI and DMABI-OH were compared with data for other ABI derivatives studied previously.
\end{abstract}

Keywords: Kerr effect, two-photon absorption, organic materials, nonlinear optics, Z-scan.

\section{INTRODUCTION}

Organic nonlinear optical (NLO) materials have showed to be promising in application for optical limiting ${ }^{1,2}$, data storage $^{3,4}$ and data processing ${ }^{5}$. Due to this increasing amount of researches are dedicated to study the third order effects such as Kerr effect and two-photon absorption as these effects are essential for the previously mentioned applications. One of the main advantages of organic materials is the possibility to tune the material properties by varying the molecular structure. At the same time the tuning can be done only after understanding all aspects of the structural and material property relation. Although extensive number of studies has been carried out to better understand structuralproperties relations, it is still insufficient for theoretically designing more efficient molecules for third order NLO applications. Previous researches have shown that highly $\pi$-conjugated molecules exhibit strong third-order NLO properties $^{6}$. Also different acceptor and donor group effect on Kerr and two-photon absorption effects have been extensively studied ${ }^{7-9}$. At the same time in vast number of studies the quantitative approach to this problem is employed in which only molecular selection is done based on results of NLO measurements. In this paper we concentrate more on structural-properties relations and study how different acceptor and donor groups affect Kerr and two-photon absorption effects.

Different experimental methods can be used to determine Kerr and two-photon absorption coefficients, such as degenerated four-wave mixing method ${ }^{10}$, nonlinear ellipse rotation method ${ }^{11}$ and Z-scan method ${ }^{12}$. Each of these methods has their advantages compared to other. For example, degenerated four-wave mixing allows to determine certain tensor elements of third-order susceptibility while the Z-scan method is easier to implement and allows to study two-photon absorption and Kerr effects simultaneously. Due to this Z-scan is one of the most popular methods for studying material third-order NLO properties.

In this paper we studied different aminobenziliden-1,3-indandione (ABI) derivatives to better understand how different chemical groups influence third-order nonlinear optical properties of organic materials. During our experiment we studied solutions where organic compounds were dissolved in chloroform. Previous studies have shown that NLO properties can depend on pulse duration ${ }^{13}$, organic solute concentration in solution ${ }^{14}$ as well as laser pulse repetition rate $^{15}$. In this work we employed $1064 \mathrm{~nm} \mathrm{Nd:YAG} \mathrm{laser} \mathrm{with} 30 \mathrm{ps}$ pulse duration and $10 \mathrm{~Hz}$ pulse repetition rate for Z-scan measurements. Our previous work showed that these experimental conditions lead to correct evaluation of NLO effect magnitude ${ }^{16}$. We also compared experimental and quantum chemical calculation values for second-order hyperpolarizability of studied materials. 


\section{EXPERIMENTAL SECTION}

In this work we studied such ABI derivatives as DDMABI and DMABI-OH (see Figure 1) and compared them to previously studied ABI derivatives described elsewhere ${ }^{16}$. We have previously studied the second-order NLO properties of these materials ${ }^{17,18}$. All studied materials belong to donor (D) and acceptor (A) molecule class and can be abbreviated in form D-A. Selected materials were chosen to study specific structure-property relations. The specific molecules were chosen to understand how third-order NLO properties change by reinforcing acceptor with dicyanomethlene group and donor group by hydroxyl group. Molar absorption spectra of studied materials dissolved in analytic grade chloroform are shown in Figure 2 with peaks at $385 \mathrm{~nm}$ for DMABI-OH and $557 \mathrm{~nm}$ for DDMABI. One of the conditions for twophoton absorption is that material has considerable linear absorption at half-wavelength. DDMABI has a considerable absorption coefficient at $532 \mathrm{~nm}$ which leads us to believe that this material could exhibit two-photon absorption.<smiles>O=C1C(=Cc2ccc(O)cc2)C(=O)c2ccccc21</smiles>

DMABI-OH

2-(4-hydroxybenzylidene)-indane-1,3-dione<smiles>CN(C)c1ccc(C=C2C(=O)c3ccccc3C2=C(C#N)C#N)cc1</smiles>

D-DMABI

2-[4-(N,N-dimethylamino)benzylidene]1 -indenone-3-ylidenepropanedinitrile

Figure 1. Structural formulas and names of studied organic molecules.

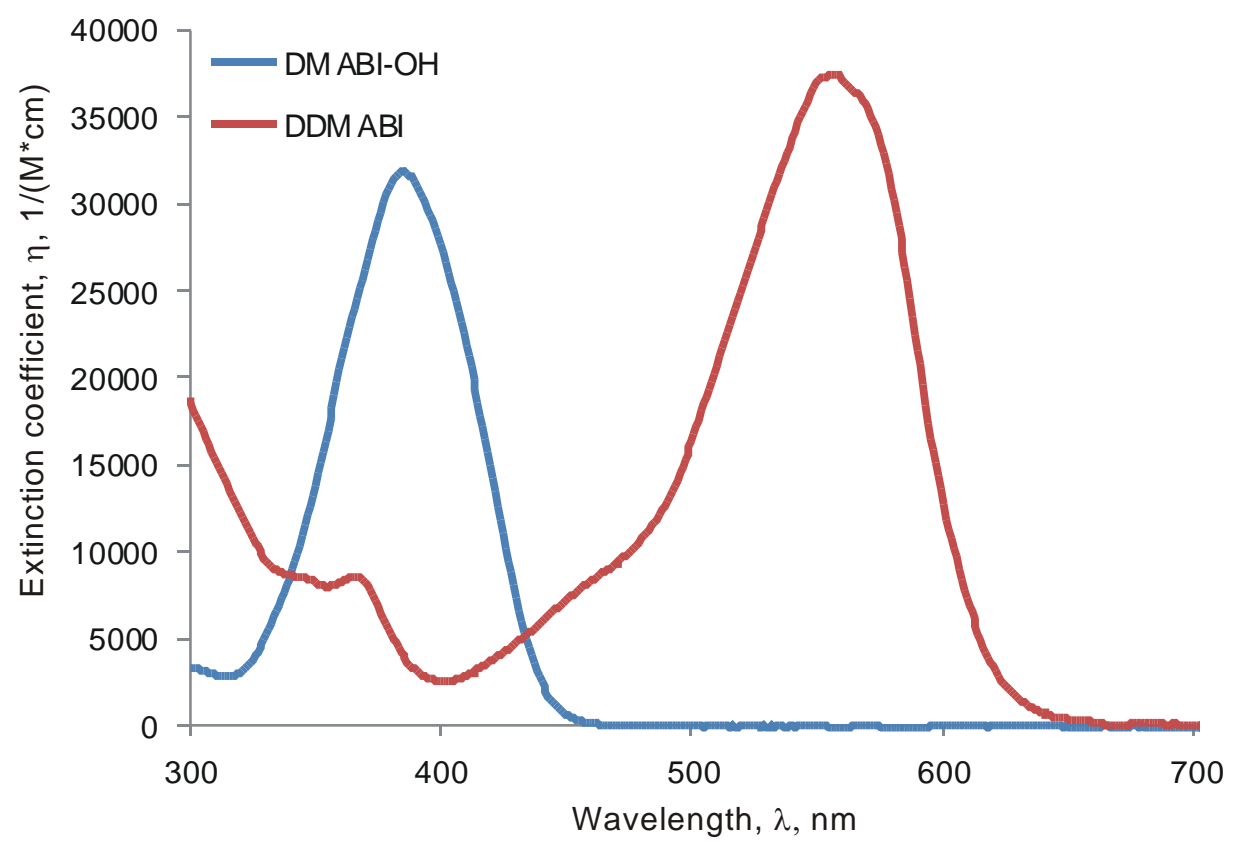

Figure 2. Molar absorption of studied molecules. As evident DDMABI shows considerable absorption at $532 \mathrm{~nm}$. 
Sample concentration was measured as organic compound mass our chloroform in percentage and varied from $0.001 \%$ to $0.05 \%$. There were two main restrictions for using samples with higher concentration. Firstly, the material has weak solubility in chloroform. Secondly the samples needed to fit weak nonlinear media model implemented in data analysis process $^{12}$. Studied samples were contained in $2 \mathrm{~mm}$ thick optical quartz cells.

The description of implemented Z-scan setup can be found in our previous paper ${ }^{16}$. Experimental parameters of Z-scan setup were as follows: for laser beam focusing we used lens with $11 \mathrm{~cm}$ focus length, beam waist radius at focus was calculated to be $w_{0}=26 \mu \mathrm{m}$ and we used $1 \mathrm{~mm}$ aperture for closed-aperture measurements which separated less than $1 \%$ of incident laser beam. These experimental parameters allowed us to use thin sample approximation for data processing $^{12}$.

To describe Kerr effect and two-photon absorption effect we used the following formalism. Refractive index $n$ changes due to Kerr effect can be expressed in the following way:

$$
n=n_{0}+n_{2} I,
$$

where $n_{0}$ is linear refractive index of the material, $n_{2}$ is nonlinear refractive index and $I$ is incident light intensity. In case of two-photon absorption we study how materials absorption $\alpha$ changes due to incident light intensity:

$$
\alpha=\alpha_{0}+\alpha_{2} I
$$

where $\alpha_{0}$ and $\alpha_{2}$ are linear and nonlinear absorption coefficients, respectively.

During Z-scan measurement we detect sample transmittance as function from sample position using two detectors - open aperture and closed aperture detectors. If two-photon absorption is present in NLO media, then sample transmittance can be expressed as follows:

$$
T(z)=\sum_{i=0}^{\infty} \frac{\frac{\alpha_{2} L_{e f f} I^{2}}{\left(1+\frac{z^{2}}{z_{0}^{2}}\right)}}{(m+1)^{\frac{3}{2}}}
$$

where $z$ is sample position relative to focal point and $L_{e f f}$ and $z_{0}$ is parameters defined by following relations:

$$
\left\{\begin{array}{c}
z_{0}=\frac{k w_{0}^{2}}{2} \\
L_{e f f}=\frac{l-e^{-\alpha L}}{\alpha},
\end{array}\right.
$$

where $k$ is the wave number, $w_{0}$ is beam waist radius at focal point and $L$ is sample thickness. This theoretical model can be used for cases when parameter $\left|\alpha_{2} L_{e f f} I\right|<1$. To better compare two-photon absorption properties of molecules it is better to use two-photon absorption cross-section as it defines single molecules two-photon absorptions properties. Twophoton absorption cross-section can be calculated by following expression:

$$
\sigma_{2 P A}=\frac{h v}{N} \alpha_{2}
$$

where $h v$ is the photon energy and $N$ is the molecule concentration per $\mathrm{cm}^{3}$. If Kerr effect is present in NLO media, then sample transmittance can be expressed as follows:

$$
T(z)=1+\frac{4 \Delta \Phi \frac{z}{z_{0}}}{\left(\frac{z^{2}}{z_{0}^{2}}+9\right)\left(\frac{z^{2}}{z_{0}^{2}}+1\right)}
$$

where $\Delta \Phi$ is phase changes detected by laser beam defined by expression:

$$
\Delta \Phi=k n_{2} I L_{e f f}
$$


Similar as for two-photon absorption, this theoretical model can be applied for case of weak nonlinear media, for which $|\Delta \Phi|<\pi$. This is essential for correct evaluation of NLO coefficients ${ }^{14}$. During experimental measurements both of the effects can be present in media. While open aperture measurements are only influenced by two-photon absorption, closed aperture measurement is influenced by both. To separate the influence of Kerr effect on transmittance we divide closed aperture data with open aperture data. Laser intensity at focal point was varied in range from 10 to $50 \mathrm{GW} / \mathrm{cm}^{2}$. Usage of higher laser intensities was limited by conditions for weak nonlinear media as well as to avoid sample degradation and two-photon excited state absorption ${ }^{19}$.

\section{RESAULTS AND DISCUSSION}

To verify the validity of our experimental setup we performed a series of tests with chloroform solution as it is widely used as reference material for third-order NLO studies. Our acquired value of Kerr effect coefficient for chloroform was measured to be $n_{2}=1.88 \pm 0.12 \cdot 10^{-15} \mathrm{~cm}^{2} / \mathrm{W}$ which fits with values presented in literature ${ }^{6}$. Next we carried out experimental measurements for DMABI-OH and DDMABI in chloroform solution. NLO contribution of solution and solute is defined by weight fraction. This allows us to calculate NLO properties of organic compounds by using following equation ${ }^{20}$ :

$$
n_{2}=(1-\rho) n_{2 ; \mathrm{CHCl}_{3}}+\rho n_{2 ; \text { solute }} \text {, }
$$

where, $\rho$ is concentration. Experimental data for DDMABI is displayed in Figure 3. To acquire values for Kerr and twophoton absorption coefficients we fitted experimental data with equations (3) and (6). Acquired values for NLO coefficients are presented in Table 1 . By comparing Kerr coefficients $n_{2 ; \text { Kerr }}$ we can see that DMABI-OH and DMABI both have positive signs. Also we can see that DDMABI poses two-photon absorption. This correlates with assumption that two-photon absorption is present for materials that possess considerable absorption at half-wavelength of laser beam.

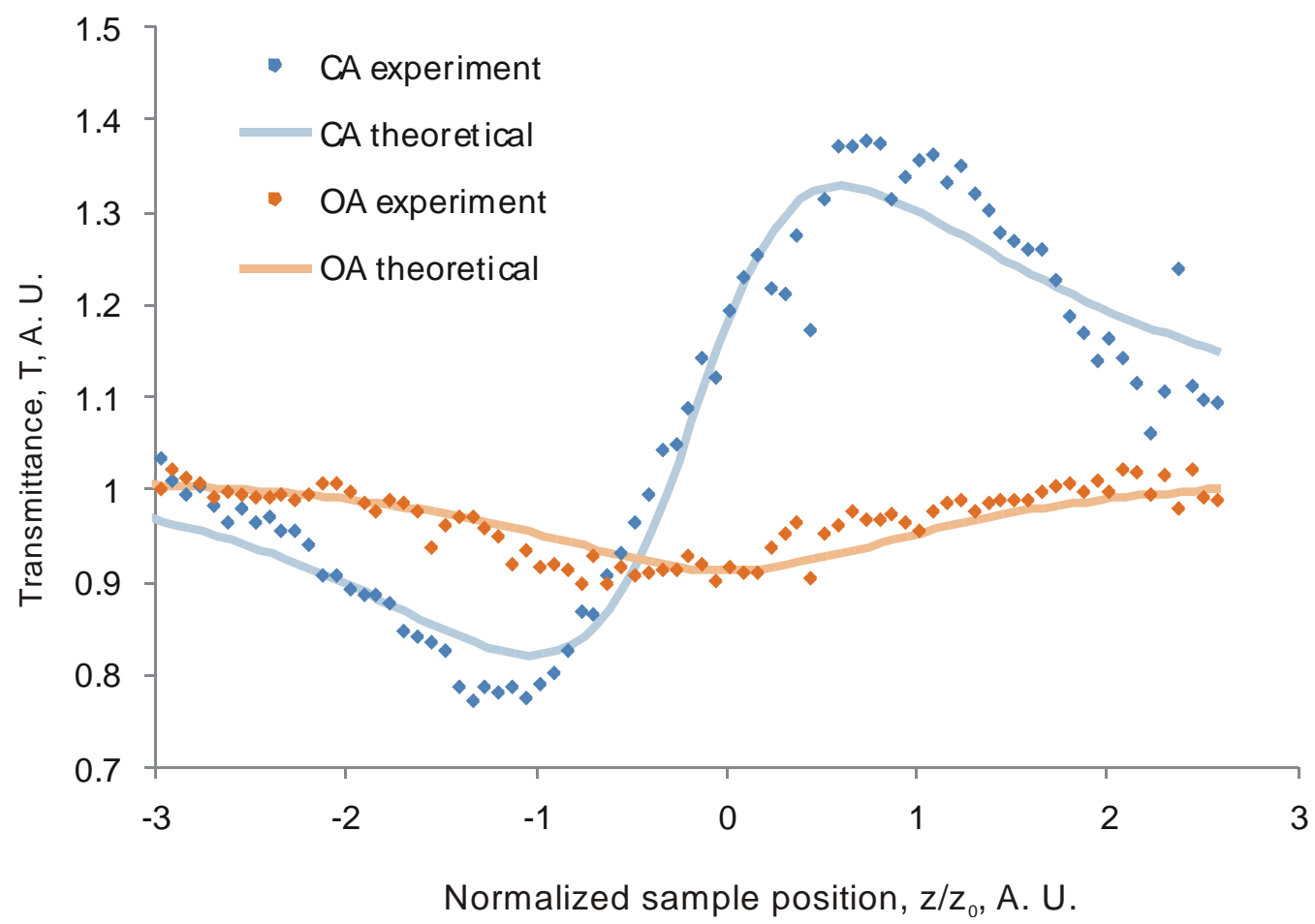

Figure 3. Z-scan experimental data for DDMABI. Laser irradiance at focal point was $42 \mathrm{GW} / \mathrm{cm}^{2}$ while sample concentration was $0.03 \%$. 
Table 1. Optical and NLO coefficients of studied components as well as DMABI studied in specified reference.

\begin{tabular}{|c|c|c|c|c|c|c|c|c|}
\hline Solute & $\begin{array}{l}\text { Extinction } \\
\text { coefficient } \\
\text { at } 532 \mathrm{~nm}\end{array}$ & $\begin{array}{c}\mathrm{M}, \\
\mathrm{g} / \mathrm{mol}\end{array}$ & $\begin{array}{c}\alpha_{2}, \mathrm{~cm} / \mathrm{W} \\
10^{-8}\end{array}$ & $\begin{array}{c}\sigma_{2 \mathrm{PA}} \\
\mathrm{GM}\end{array}$ & $\begin{array}{c}\mathrm{n}_{2 ; \mathrm{Kerr}}, \mathrm{cm}^{2} / \mathrm{W} \\
\cdot 10^{-12}\end{array}$ & $\begin{array}{c}\chi_{\mathrm{Re},} \\
\mathrm{m}^{2} / \mathrm{V}^{2} \cdot 10^{-19}\end{array}$ & $\begin{array}{c}\begin{array}{c}\chi_{\operatorname{Im}}, \\
\mathrm{m}^{2} / \mathrm{V}^{2} \cdot 10^{-} \\
19\end{array} \\
\end{array}$ & $\gamma$, esu $\cdot 10^{-34}$ \\
\hline Chloroform & - & 119.37 & - & - & $\begin{array}{c}0.00188 \\
\pm 0.00012\end{array}$ & $\begin{array}{c}0.01392 \\
\pm 0.00089\end{array}$ & - & $\begin{array}{c}0.0181 \\
\pm 0.0011\end{array}$ \\
\hline DMABI-OH & - & 250.25 & - & - & $2.75 \pm 0.34$ & $28.6 \pm 3.5$ & - & $78.1 \pm 9.7$ \\
\hline DDMABI & 30458 & 325.37 & $9.62 \pm 0.89$ & $652 \pm 60$ & $0.339 \pm 0.043$ & $3.52 \pm 0.45$ & $8.64 \pm 0.80$ & $12.5 \pm 1.6$ \\
\hline DMABI $^{16}$ & 1023 & 277.33 & - & - & $0.249 \pm 0.022$ & $2.59 \pm 0.23$ & - & $7.83 \pm 0.69$ \\
\hline
\end{tabular}

To calculate values for real and imaginary parts of third-order susceptibility of our materials we used formulas presented in literature ${ }^{21}$ :

$$
\begin{aligned}
& \chi_{R e}^{(3)}=\left(4 \cdot \frac{\varepsilon_{0} \cdot c \cdot n_{0}^{2}}{3}\right) \cdot n_{2}\left(\frac{m^{2}}{W}\right), \\
& \chi_{I m}^{(3)}=\left(\frac{\varepsilon_{0} \cdot c \cdot n_{0}^{2} \cdot \lambda}{3 \cdot \pi}\right) \cdot \alpha_{2}\left(\frac{m}{W}\right),
\end{aligned}
$$

where $\varepsilon_{0}$ is vacuum dielectric constant, $c$ is speed of light in vacuum and $\lambda$ is laser wavelength. To better compare NLO properties on molecular scale we calculated values for second-order hyperpolarizabilty of our materials using following expression $^{22}$ :

$$
\gamma=\frac{\chi^{(3)}}{\left[\frac{4}{3}\left(n_{0}^{2}+2\right)\right]_{N}^{4}},
$$

where $N$ is molecule concentration per $\mathrm{cm}^{3}$. Values for all of the coefficients are represented in Table 1 . We also carried out quantum chemical calculations for second-order hyperpolarizability (see Table 2). All of the parameters for calculations can be found in our previous work ${ }^{16}$. Experimental and calculated values for second-order hyperpolarizability of molecules studied in this work and our previous work are shown in Figure 4. Correlation between both values can be seen for most of the organic compounds. DMABI-OH shows larger difference for calculated and experimental values then rest of the molecules. This could be due photo-chemical processes present during measurement.

Table 2. Second-order hyperpolarizability values for studied components as well as DMABI studied in specified reference.

\begin{tabular}{ccc} 
Solute & $\gamma_{\text {exp }}$ esu $\cdot 10^{-34}$ & $\gamma_{\text {calc }}$ esu $\cdot 10^{-34}$ \\
\hline \hline Chloroform & $0.0181 \pm 0.0011$ & 0.0245 \\
DMABIOH & $78.1 \pm 9.7$ & 1.37 \\
DDMABI & $12.5 \pm 1.6$ & 4.67 \\
DMABI $^{16}$ & $7.83 \pm 0.69$ & 2.97
\end{tabular}




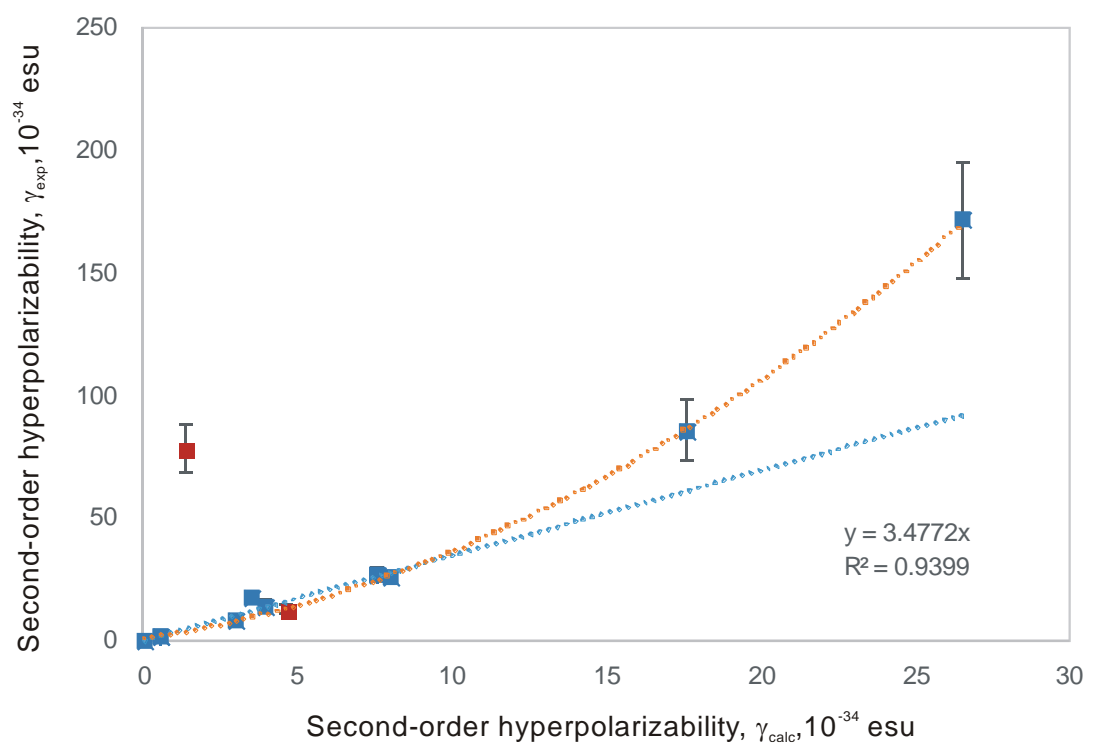

Figure 4. Experimental values for second-order hyperpolarizability $\gamma_{\exp }$ displays linear correlation with quantum calculated values $\gamma_{\text {calc }}$ for most of the molecules. Blue dots are values from reference ${ }^{16}$ and red dots are values acquired in this work.

By comparing NLO coefficients of studied components with previously results for DMABI, we can conclude that by adding dicyanomethlene group to acceptor we increase the amplitude of Kerr effect. This correlates with assumption that the strength of acceptor and donor groups influences the amplitude of Kerr effect. Also we can see that the hydroxyl group, similar to dimethylamino group for DMABI, gives positive sign to Kerr effect.

\section{CONCLUSIONS}

In this paper we studied how specific molecular alterations influence NLO properties of organic materials. We evaluated the magnitude of Kerr and two-photon absorption effects for DDMABI and DMABI-OH in chloroform and compered them to previously studied ABI derivatives. Both of the molecules possess positive sign for Kerr effect, but only for DDMABI we observed two-photon absorption. This is because only DDMABI possess considerable absorption at 532 $\mathrm{nm}$. By comparing both of these molecules to DMABI we concluded that i) dicyanomethlene group increases magnitude of Kerr effect, ii) hydroxyl group, as well as dimethylamino group in DMABI, possess positive sign for Kerr effect. By comparing experimental and quantum chemical calculation values of second-order hyperpolarizability we can see that for most molecules calculations allow to predict which molecules will possess Kerr effect with larger or smaller magnitude. DMABI-OH displays inadequacy with this assumption. This could be due to some photo-chemical processes present during measurement. Further research is necessary to prove this hypothesis.

\section{ACKNOWLEDGMENT}

This material is based upon work supported by the ERDF 1.1.1.1 activity project Nr. 1.1.1.1/16/A/046 "Application assessment of novel organic materials by prototyping of photonic devices" as well as by National Research Program "Multifunctional Materials and Composites, Photonics and Nanotechnology" (IMIS2) project "Photonics and materials for photonics". 


\section{REFERENCES}

[1] Borshch, A. A., Brodyn, M. S., Starkov, V. N., Rudenko, V. I., Volkov, V. I., Boyarchuk, A. Y.., Semenov, A. V., "Broadband optical limiting in thin nanostructured silicon carbide films and its nature," Opt. Commun. 364, 88-92, Elsevier (2016).

[2] Ma, H., Leng, J., Liu, M., Zhao, L.., Jiao, Y., "Two-photon absorption and optical limiting of a fluorenyl-based chromophore with femtosecond laser pulse," Opt. Commun. 350, 144-147, Elsevier (2015).

[3] Li, L., Wang, P., Hu, Y., Lin, G., Wu, Y., Huang, W.., Zhao, Q., "Novel carbazole derivatives with quinoline ring: Synthesis , electronic transition, and two-photon absorption three-dimensional optical data storage," Spectrochim. ACTA PART A Mol. Biomol. Spectrosc. 139, 243-252, Elsevier B.V. (2015).

[4] Hu, D., Hu, Y., Huang, W.., Zhang, Q., "Two-photon induced data storage in hydrogen bonded supramolecular azopolymers," Opt. Commun. 285(24), 4941-4945, Elsevier (2012).

[5] Ahadi, S.., Granpayeh, N., "Femtosecond all-optical switching based on asymmetric plasmonic Kerr Fabry-Perot resonators," Opt. Commun. 349, 36-41, Elsevier (2015).

[6] Cassano, T., Tommasi, R., Ferrara, M., Babudri, F., Farinola, G. M.., Naso, F., "Substituent-dependence of the optical nonlinearities in poly(2,5-dialkoxy-p-phenylenevinylene) polymers investigated by the Z-scan technique," Chem. Phys. 272(1), 111-118 (2001).

[7] Webster, S., Fu, J., Padilha, L. A., Przhonska, O. V., Hagan, D. J., Stryland, E. W. Van., Bondar, M. V., Slominsky, Y. L.., Kachkovski, A. D., "Comparison of nonlinear absorption in three similar dyes : Polymethine, squaraine and tetraone," Chem. Phys. 348, 143-151 (2008).

[8] Padilha, L. A., Webster, S., Przhonska, O. V., Hu, H., Peceli, D., Rosch, J. L., Bondar, M. V., Gerasov, A. O., Kovtun, Y. P., et al., "Nonlinear absorption in a series of Donor - p - Acceptor cyanines with different conjugation lengths $\dagger$," J. Mater. Chem. 19, 7503-7513 (2009).

[9] Hong, J., Przhonska, O. V., Khodja, S., Yang, S., Ross, T. S., Hagan, D. J., Stryland, E. W. Van., Bondar, M. V., Slominsky, Y. L., "Polymethine and squarylium molecules with large excited-state absorption," Chem. Phys. 245, 79-97 (1999).

[10] Kityk, I. V., Fahmi, A., Sahraoui, B., Rivoire, G.., Feeks, I., "Nitrobenzene as a material for the fast-respond degenerate four-wave mixing," Opt. Mater. (Amst). 16, 417-429 (2001).

[11] Liu, Z., Yan, X., Tian, J., Zhou, W.., Zang, W., "Nonlinear ellipse rotation modified Z-scan measurements of third-order nonlinear," Opt. Express 15(20), 13351-13359 (2007).

[12] Bahae, M. S., Said, a a., Wei, T. H., Hagan, D. J.., Stryland, E. W. Van., "Sensitive Measurements of Optical Nonlinearities Using a Single Beam,” IEEE J. Quantum Electron. 26(4), 760-769 (1990).

[13] Shinkawa, K.., Ogusu, K., "Pulse-width dependence of optical nonlinearities in As2Se3 chalcogenide glass in the picosecond-to-nanosecond region.," Opt. Express 16(22), 18230-18240 (2008).

[14] Ajami, A., Gruber, P., Tromayer, M., Husinsky, W., Stampfl, J., Liska, R.., Ovsianikov, A., "Evidence of concentration dependence of the two-photon absorption cross section: Determining the 'true' cross section value," Opt. Mater. (Amst). 47, 524-529, Elsevier B.V. (2015).

[15] Falconieri, M.., Salvetti, G., "Simultaneous measurement of pure-optical and thermo-optical nonlinearities induced by high-repetition-rate, femtosecond laser pulses: Application to CS2," Appl. Phys. B Lasers Opt. 69(2), 133-136 (1999). 
[16] Bundulis, A., Nitiss, E., Mihailovs, I., Busenbergs, J.., Rutkis, M., "Study of Structure-Third-Order Susceptibility Relation of Indandione Derivatives,” J. Phys. Chem. C, acs.jpcc.6b07003 (2016).

[17] Rutkis, M., Vembris, A., Zauls, V., Tokmakovs, A., Fonavs, E., Jurgis, A.., Kampars, V., "Novel second-order non-linear optical polymer materials containing indandione derivativatives as a chromophore," Org. Optoelectron. Photonics II 6192, 61922Q-61922Q-8 (2006).

[18] Nitiss, E., Bundulis, A., Tokmakov, A., Busenbergs, J., Linina, E.., Rutkis, M., "Review and comparison of experimental techniques used for determination of thin film electro-optic coefficients," Phys. Status Solidi Appl. Mater. Sci. 212(9), 1867-1879 (2015).

[19] Gu, B., Ji, W., Patil, P. S., Dharmaprakash, S. M.., Wang, H.-T., "Two-photon-induced excited-state absorption: Theory and experiment," Appl. Phys. Lett. 92(9), 91118 (2008).

[20] Samoc, M., Samoc, A.., Luther-davies, B., "Femtosecond Z-scan and degenerate four-wave mixing measurements of real and imaginary parts of the third-order nonlinearity of soluble conjugated polymers," J. Opt. Soc. Am. B 15(2), 817-825 (1998).

[21] del Coso, R.., Solis, J., "Relation between nonlinear refractive index and third-order susceptibility in absorbing media,” J. Opt. Soc. Am. B 21(3), 640 (2004).

[22] Sharafudeen, K. N., Adithya, A., Vijayakumar, S., Sudheesh, P., Kalluraya, B.., Chandrasekharan, K., "Multiphoton absorption process and self-focusing effect in coumarin derivative doped PMMA films by Z-scan and optical limiting studies," Curr. Appl. Phys. 11(4), 1089-1093, Elsevier B.V (2011).

Institute of Solid State Physics, University of Latvia as the Center of Excellence has received funding from the European Union's Horizon 2020 Framework Programme H2020-WIDESPREAD-01-2016-2017-TeamingPhase2 under grant agreement No. 739508, project CAMART² 